\title{
Predictive Value of Tertiary Lymphoid Structures Assessed by High Endothelial Venule Counts in the Neoadjuvant Setting of Triple-Negative Breast Cancer
}

\author{
In Hye Song, $M D^{1}$ \\ Sun-Hee Heo, $\mathrm{PhD}^{1,2}$ \\ Won Seon Bang, BS \\ Hye Seon Park, BS \\ In Ah Park, MD' \\ Young-Ae Kim, PhD ${ }^{1,2}$ \\ Suk Young Park, MS \\ Jin Roh, MD 1 \\ Gyungyub Gong, MD, $\mathrm{PhD}$ \\ Hee Jin Lee, MD, PhD ${ }^{1}$
}

\begin{abstract}
${ }^{1}$ Department of Pathology, Asan Medical Center, University of Ulsan College of Medicine, Seoul, ${ }^{2}$ Asan Institute for Life Sciences, Asan Medical Center, University of Ulsan College of Medicine, Seoul, Korea
\end{abstract}

\author{
Correspondence: Hee Jin Lee, MD, PhD \\ Department of Pathology, Asan Medical Center, \\ University of Ulsan College of Medicine, \\ 88 Olympic-ro 43-gil, Songpa-gu, \\ Seoul 05505, Korea \\ Tel: 82-2-3010-5889 \\ Fax: 82-2-472-7898 \\ E-mail: backlila@gmail.com \\ Received May 24, 2016 \\ Accepted June 30, 2016 \\ Published Online July 27, 2016 \\ *In Hye Song and Sun-Hee Heo contributed \\ equally to this work.
}

\begin{abstract}
Purpose
The tertiary lymphoid structure (TLS) is an important source of tumor-infiltrating lymphocytes (TILS), which have a strong prognostic and predictive value in triple-negative breast cancer (TNBC). A previous study reported that the levels of CXCL13 mRNA expression were associated with TLSs, but measuring the gene expression is challenging in routine practice. Therefore, this study evaluated the MECA79-positive high endothelial venule (HEV) densities and their association with the histopathologically assessed TLSs in biopsy samples. In addition, the relationship of TLSs with the CXCL13 transcript levels and clinical outcomes were examined.
\end{abstract}

\section{Materials and Methods}

A total of 108 TNBC patients treated with neoadjuvant chemotherapy (NAC) were studied. The amounts of TILs and TLSs were measured histopathologically using hematoxylin and eosin-stained slides. The HEV densities and TIL subpopulations were measured by immunohistochemistry for MECA79, CD3, CD8, and CD20. CXCL13 mRNA expression levels using a NanoString assay (NanoString Technologies).

\section{Results}

The mean number of HEVs in pre-NAC biopsies was 12 (range, 0 to 72). The amounts of TILs and TLSs, HEV density, and CXCL13 expression showed robust correlations with each other. A lower pre-NAC clinical T stage, higher TIL and TLS levels, a higher HEV density, CD20positive cell density, and CXCL13 expression were significant predictors of a pathologic complete response (pCR). Higher CD8-positive cell density and levels of CXCL13 expression were significantly associated with a better disease-free survival rate.

\section{Conclusion}

MECA79-positive HEV density in pre-NAC biopsies is an objective and quantitative surrogate marker of TLS and might be a valuable tool for predicting pCR of TNBC in routine pathology practice.

\section{Introduction}

Recently, tumor-infiltrating lymphocytes (TILs) have been strongly associated with the anti-tumor immunity and have shown prognostic and predictive significance in a range of types of solid neoplasms [1-4]. Triple-negative breast cancers (TNBCs) show the poorest prognosis among all breast cancer

\section{Key words}

Triple-negative breast neoplasms, Immunohistochemistry, Tertiary lymphoid structure, Prognosis 
mechanism whereby lymphoid cells infiltrate tumors and interact with malignant cells might facilitate the development of more efficient immunotherapy.

Tertiary lymphoid structures (TLSs) are one of the major sources of TILs. TLSs are ectopic, vascularized lymphoid formations that can be found in inflamed or tumoral tissues [7]. In breast cancers, TLSs can be observed mainly in areas that surround the tumor and adjacent normal terminal ductal lobular units [8]. The mechanism whereby TLSs are formed is not known, but the roles for high endothelial venules (HEVs) and the chemokine CXCL13 have been suggested [9]. The HEVs are specialized blood vessels with plump and cuboidal endothelial cells, which express the peripheral node addressin (PNAd) and facilitate the extravasation of lymphocytes from the bloodstream and formation of TLSs [10]. Many studies have reported positive correlations between the TLS components, particularly HEV, and favorable clinical outcomes in various types of cancer, such as cutaneous melanoma, non-small cell lung cancer, colorectal carcinoma, and breast carcinoma [7,11-13]. Gu-Trantien et al. [14] reported that the infiltration of CXCL13-producing $\mathrm{CD}^{+}$follicular helper T cells and a CXCL13 gene expression signature are related to a longer disease-free survival in breast cancer patients. A previous study recently suggested that increased CXCL13 gene expression is predictive of a more frequent pathologic complete response (pCR) and a good prognosis in TNBC patients treated with NAC [15].

Despite the clinical significance of TLSs and TILs, they are difficult to assess in situ. The term TLS can only be used when the structure exhibits all of the following features: distinct and adjacent $\mathrm{T}$ and $\mathrm{B}$ cell compartments, fibroblastic reticular cells, HEVs, activation-induced cytidine deaminase enzyme expression, and follicular dendritic cells [16]. On the other hand, in clinical practice, it can be difficult to distinguish simple lymphoid aggregations from true TLSs using histopathological assessments alone. MECA79, which is an epitope binding to CD62L and induces TLS formation, can be used to evaluate TLS, but there has been no trial using MECA79 in tumor tissues [10]. In addition, measurements of TILs do not allow for the differentiation of various subpopulations, and there has been no consistent evidence regarding which TIL subpopulation(s) play the dominant role in anti-tumor immunity and are associated with good clinical outcomes. Some papers have reported that the presence of CD8-positive cytotoxic $\mathrm{T}$ cells is the most important predictive and prognostic factor in breast cancer $[17,18]$, whereas others have underscored the significance of B cells $[19,20]$.

Therefore, this study evaluated the amount of TLSs in preNAC core needle biopsies of TNBC by calculating the MECA79-positive HEV density, measuring the TIL subpopulations by immunohistochemistry (IHC), and using a digital computer analyzer, and assessed their relationship with
CXCL13 mRNA expression and the clinical outcomes.

\section{Materials and Methods}

\section{Patients}

A total of 108 patients, who were diagnosed with primary TNBC, received anthracycline and taxane-based NAC, and were treated surgically between 2010 and 2012 at the Asan Medical Center, were enrolled in this study. All patients received anthracycline and taxane-based regimens, which included four cycles of $60 \mathrm{mg} / \mathrm{m}^{2}$ adriamycin and 600 $\mathrm{mg} / \mathrm{m}^{2}$ cyclophosphamide followed by four cycles of 75 $\mathrm{mg} / \mathrm{m}^{2}$ docetaxel. Surgery was performed $\sim 3-4$ weeks after the last chemotherapy cycle. The occurrence of pCR was defined as the absence of a residual invasive carcinoma in the breast and regional lymph nodes (ypT0/Tis, N0). Approval of the study protocol was granted by the Institutional Review Board of the Asan Medical Center (approval number: 2013-0866).

The patients' medical records were reviewed to gather clinical information. The histological parameters were evaluated based on hematoxylin and eosin (H\&E)-stained slides and pathology reports. The clinicopathological parameters evaluated in each case included the patients' age at diagnosis, sex, tumor size, histological subtype, histological grade, pathologic tumor (pT) stage, lymphovascular invasion, lymph node metastasis, overall tumor stage, the presence or absence of recurrence, the most recent follow-up date, and the survival status.

\section{Histological evaluations}

The entire tumor bed was submitted for a pathological evaluation. H\&E-stained slides of the pre-NAC biopsy and post-NAC surgery samples of all 108 tumors were reviewed for a histological diagnosis, subtype and grade, tumor size, ypT stage, ypN stage, lymphovascular invasion, and responsiveness to chemotherapy. The histological type was defined based on the 2012 World Health Organization classification criteria, and the histological grade was assessed using the modified Bloom-Richardson classification. The Miller-Payne grade and residual cancer burden (RCB) were also assessed for their response to chemotherapy [21]. Pre-NAC biopsy slides were analyzed histopathologically to determine the level of TILs (this is defined as the mean percentage of stroma of an invasive carcinoma infiltrated by lymphocytes and plasma cells in $10 \%$ increments; $1 \%$ or $5 \%$ criteria were used if less than $10 \%$ of stroma was infiltrated by TILs; all 
available full sections were evaluated) [22], the TLSs in the adjacent tissues, including carcinoma in situ components (none, little, moderate, or abundant), and the presence or absence of a germinal center in TLSs. Lymphoid aggregation with vessels that exhibited HEV-like features (plump, cuboidal endothelial cells) with or without germinal centers was considered to be a TLS.

\section{Immunohistochemical evaluations}

Among the 108 cases, formalin-fixed paraffin-embedded (FFPE) tissue samples were available for 55 cases, which were analyzed by IHC and mRNA expression. The FFPE tissue sections were stained using an automatic immunohistochemical staining device (Benchmark XT, Ventana Medical

Table 1. Clinicopathologic characteristics of the tumors according to the availability of FFPE tumor blocks

\begin{tabular}{|c|c|c|c|}
\hline Parameter & $\begin{array}{l}\text { Tumors without available } \\
\text { FFPE blocks }(n=53)\end{array}$ & $\begin{array}{l}\text { Tumors with available } \\
\text { FFPE blocks }(n=55)\end{array}$ & p-value \\
\hline Age (yr) & $43.9 \pm 10.2$ & $41.4 \pm 9.6$ & 0.181 \\
\hline \multicolumn{4}{|c|}{ Clinical tumor stage } \\
\hline $\mathrm{T} 1$ & $4(7.5)$ & $4(7.3)$ & 0.942 \\
\hline $\mathrm{T} 2$ & $27(50.9)$ & $31(56.4)$ & \\
\hline T3 & $19(35.8)$ & $18(32.7)$ & \\
\hline $\mathrm{T} 4$ & $3(5.7)$ & $2(3.6)$ & \\
\hline \multicolumn{4}{|c|}{ Clinical nodal status } \\
\hline N0 & $5(9.4)$ & $1(1.8)$ & 0.331 \\
\hline N1 & $21(39.6)$ & $27(49.1)$ & \\
\hline N2 & $12(22.6)$ & $11(20.0)$ & \\
\hline N3 & $15(28.3)$ & $16(29.1)$ & \\
\hline \multicolumn{4}{|c|}{ Histologic grade } \\
\hline 2 & $24(45.3)$ & $25(45.5)$ & 1 \\
\hline 3 & $29(54.7)$ & $30(54.5)$ & \\
\hline TIL $(\%)$ & $23.3 \pm 20.6$ & $25.9 \pm 21.0$ & 0.471 \\
\hline \multicolumn{4}{|l|}{ TLS } \\
\hline None & $9(22.5)$ & $6(13.0)$ & 0.198 \\
\hline Mild & $12(30.0)$ & 9 (19.6) & \\
\hline Moderate & $14(35.0)$ & $18(39.1)$ & \\
\hline Abundant & $5(12.5)$ & $13(28.3)$ & \\
\hline \multicolumn{4}{|c|}{ Germinal center in TLSs } \\
\hline Absent & $36(97.3)$ & $44(95.7)$ & 1 \\
\hline Present & $1(2.7)$ & $2(4.3)$ & \\
\hline \multicolumn{4}{|l|}{ pCR } \\
\hline Absent & $37(69.8)$ & $38(69.1)$ & 1 \\
\hline Present & $16(30.2)$ & $17(30.9)$ & \\
\hline \multicolumn{4}{|c|}{ Miller-Payne grade } \\
\hline Grade 1 & $1(1.9)$ & 0 & 0.115 \\
\hline Grade 2 & $2(3.8)$ & $10(18.2)$ & \\
\hline Grade 3 & $18(34.0)$ & $14(25.5)$ & \\
\hline Grade 4 & $12(22.6)$ & $13(23.6)$ & \\
\hline Grade 5 & $20(37.7)$ & $18(32.7)$ & \\
\hline \multicolumn{4}{|l|}{ RCB class } \\
\hline 0 & $16(30.2)$ & $17(30.9)$ & 0.295 \\
\hline 1 & $5(9.4)$ & $1(1.8)$ & \\
\hline 2 & $20(37.7)$ & $27(49.1)$ & \\
\hline 3 & $12(22.6)$ & $10(18.2)$ & \\
\hline
\end{tabular}

Values are presented as mean \pm standard deviation or number (\%). FFPE, formalin-fixed paraffin-embedded; TIL, tumor-infiltrating lymphocytes; TLS, tertiary lymphoid structure; pCR, pathologic complete response; RCB, residual cancer burden. 
Systems, Tucson, AZ). The antibodies to CD3 (1:50, Novocastra Laboratories, Newcastle upon Tyne, UK), CD8 (1:400, Dako, Glostrup, Denmark), CD20 (1:500, Novocastra Laboratories), and MECA79 (1:200, Santa Cruz Biotechnology, Dallas, TX; recognizes sulfate-dependent carbohydrate epitopes of PNAd expressed in endothelial cells of HEVs), were used. The total number of MECA79-positive blood vessels in each slide were counted manually.

The immunostained slides were scanned using a digital microscopy scanner (Pannoramic 250 FLASH, 3DHISTECH Ltd., Budapest, Hungary). The entire tumor area was selected in whole slides. In each case, the images were then scanned using computer viewer software (Pannoramic Viewer 1.15.2, 3DHISTECH Ltd.) and the number of CD3-, CD8-, and CD20-positive lymphocytes were counted in the tumor area using the NuclearQuant module. The positive cell densities were calculated by dividing the immuno-positive cell numbers by the tumor area.

\section{Gene expression analysis using a NanoString nCounter system}

The levels of the CXCL13 mRNA transcripts in the FFPE tissue samples were evaluated by digital transcript counting (nCounter GX Human Immunology V2 Kit) assay (NanoString Technologies, Seattle, WA) [23]. The total RNA (100 ng) was assayed on a Counter Digital Analyzer (NanoString Technologies) according to the manufacturer's instructions. The data were normalized by scaling with the geometric mean of the built-in control gene probes for each sample. Finally, a log transformation (base 2) was performed.

\section{Statistical analysis}

All statistical analyses were performed using the SPSS ver. 18 (SPSS Inc., Chicago, IL). Wilcoxon signed-rank tests, Kruskal-Wallis tests, Mann-Whitney U tests, Fisher exact tests, logistic regression, Spearman's correlations, and binary logistic regression were used where appropriate. A threshold for statistical significance was set to $5 \%$.

\section{Results}

\section{Clinicopathological characteristics of the study cases}

The patients' ages ranged from 23 to 70 years (median, 42 years); all patients were women. Among the 108 cases analyzed, eight were cT1 tumors, 58 were cT2 tumors, 37 were cT3 tumors, and five were cT4 tumors. For the nodal status,

Table 2. Predictive values of TIL, TLS, and other clinicopathological parameters prior to neoadjuvant chemotherapy for a pathologic complete response in 108 cases

\begin{tabular}{|c|c|c|c|c|c|c|}
\hline \multirow{2}{*}{ Parameter } & \multicolumn{3}{|c|}{ Univariate analysis } & \multicolumn{3}{|c|}{ Multivariate analysis } \\
\hline & HR & $95 \% \mathrm{CI}$ & p-value & HR & $95 \% \mathrm{CI}$ & p-value \\
\hline Clinical tumor stage & & & 0.067 & & & 0.304 \\
\hline \multicolumn{7}{|l|}{$\mathrm{T} 1$} \\
\hline $\mathrm{T} 2$ & 0.150 & $0.028-0.816$ & 0.028 & 0.294 & $0.046-1.862$ & 0.193 \\
\hline T3 & 0.092 & $0.015-0.546$ & 0.009 & 0.147 & 0.020-1.094 & 0.061 \\
\hline $\mathrm{T} 4$ & 0.083 & $0.006-1.257$ & 0.073 & 0.289 & $0.015-5.418$ & 0.406 \\
\hline Clinical nodal status & & & 0.690 & & & - \\
\hline \multicolumn{7}{|l|}{ N0 } \\
\hline N1 & 1.097 & $0.182-6.618$ & 0.920 & - & - & - \\
\hline N2 & 0.875 & $0.129-5.943$ & 0.891 & - & - & - \\
\hline N3 & 0.583 & $0.088-3.880$ & 0.577 & - & - & - \\
\hline Histologic grade (3 vs. 2 ) & 1.704 & $0.735-3.953$ & 0.214 & - & - & - \\
\hline TIL in biopsies & 1.025 & $1.005-1.046$ & 0.016 & 1.038 & $1.012-1.065$ & 0.004 \\
\hline TLS in biopsies & & & 0.147 & & & 0.925 \\
\hline \multicolumn{7}{|l|}{ None } \\
\hline Little & 2.031 & $0.337-12.24$ & 0.439 & 1.086 & $0.158-7.472$ & 0.933 \\
\hline Moderate & 2.955 & $0.559-15.63$ & 0.202 & 1.478 & $0.248-8.815$ & 0.668 \\
\hline Abundant & 6.500 & $1.127-37.48$ & 0.036 & 1.721 & $0.213-13.92$ & 0.611 \\
\hline
\end{tabular}

TIL, tumor-infiltrating lymphocytes; TLS, tertiary lymphoid structure; HR, hazard ratio; CI, confidence interval. 
Table 3. Disease-free survival analyses based on TIL, TLS, and clinicopathological parameters in 108 cases

\begin{tabular}{|c|c|c|c|c|c|c|}
\hline \multirow{2}{*}{ Parameter } & \multicolumn{3}{|c|}{ Univariate analysis } & \multicolumn{3}{|c|}{ Multivariate analysis } \\
\hline & HR & $95 \% \mathrm{CI}$ & p-value & HR & $95 \% \mathrm{CI}$ & p-value \\
\hline cT stage (T3-4 vs. T1-2) & 2.335 & $1.252-4.355$ & 0.008 & 1.877 & $0.923-3.813$ & 0.082 \\
\hline cN stage (N2-3 vs. N0-1) & 1.652 & $0.881-3.096$ & 0.117 & 1.722 & $0.847-3.501$ & 0.133 \\
\hline Histologic grade (3 vs. 2) & 0.796 & $0.427-1.483$ & 0.473 & & & \\
\hline TIL in biopsies & 0.975 & $0.957-0.993$ & 0.007 & 0.975 & 0.954-0.997 & 0.028 \\
\hline $\begin{array}{l}\text { TLS in biopsies } \\
\text { (abundant vs. none to moderate) }\end{array}$ & 0.327 & $0.100-1.074$ & 0.065 & 0.624 & $0.164-2.374$ & 0.489 \\
\hline
\end{tabular}

TIL, tumor-infiltrating lymphocytes; TLS, tertiary lymphoid structure; HR, hazard ratio; CI, confidence interval.

A
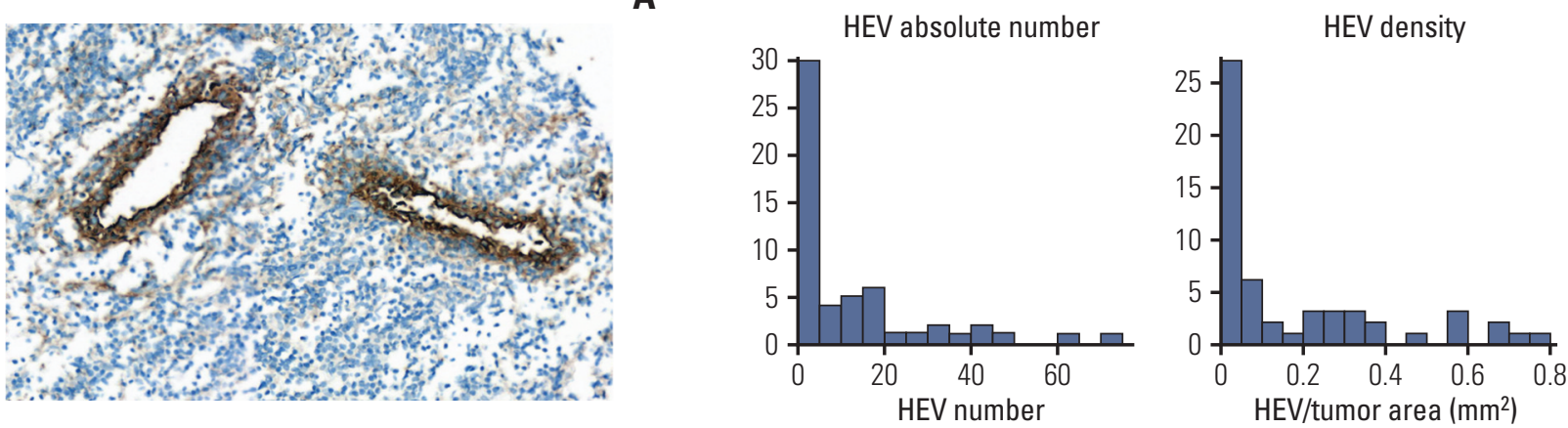

Fig. 1. High endothelial venules (HEVs) in needle biopsy specimens of triple-negative breast cancer. (A) Representative microscopic images of HEVs immunohistochemically stained with MECA79 (×400). (B) Absolute number and density of HEVs in tumors $(n=55)$.

there were six cN0 tumors, $48 \mathrm{cN} 1$ tumors, $23 \mathrm{cN} 2$ tumors, and $31 \mathrm{cN} 3$ tumors. For staging, 37 and 71 were clinical stage II and III, respectively. pCR was achieved in 33 of 108 tumors (30.6\%). The median patient follow-up duration was 34.9 months (range, 12.0 to 55.8 months). The mean TIL level was $24.6 \pm 20.8(\%)$. Table 1 summarizes the clinicopathological characteristics of the tumors without and with available FFPE blocks. No significant differences were observed between the two groups regarding the patients' age, clinical T or N stage, histological grade, TILs, TLSs, pCR rate, MillerPayne grade, or RCB class.

\section{Predictive value and prognostic significance of TILs and TLSs}

Based on assessments of the H\&E-stained sections, uniand multi-variate analyses were performed to assess the associations between PCR, TIL, and other established clinicopathological parameters (Table 2). Among the 108 tumors, univariate logistic regression analysis revealed a lower cT stage and higher TIL and TLS levels in the pre-NAC biopsy to be significantly associated with pCR. Multivariate analysis, including the cT stage, TIL, and TLS, showed that only TIL (hazard ratio [HR], 1.038; 95\% confidence interval [CI], 1.012 to $1.065 ; \mathrm{p}=0.004$ ) was an independent predictor of $\mathrm{pCR}$. In univariate disease-free survival analysis, the cT and TIL were closely associated with a longer disease-free survival rate (Table 3). On the other hand, the TLS level was not significantly associated with the patients' survival. Multivariate analysis, including the cT stage, cN stage, TIL, and TLS, showed that only TIL (HR, 0.975; 95\% CI, 0.954 to 0.997; $\mathrm{p}=0.028$ ) was a robust independent prognostic factor for the disease-free survival.

\section{Correlations of various parameters associated with TILs}

IHC and gene expression analyses were carried out in 55 cases with available FFPE tissue blocks. The median of the tissue areas in each slide was $69.46 \mathrm{~mm}^{2}$ (range, 23.47 to $175.33 \mathrm{~mm}^{2}$ ), whereas the median of the tumor areas was 
CD3
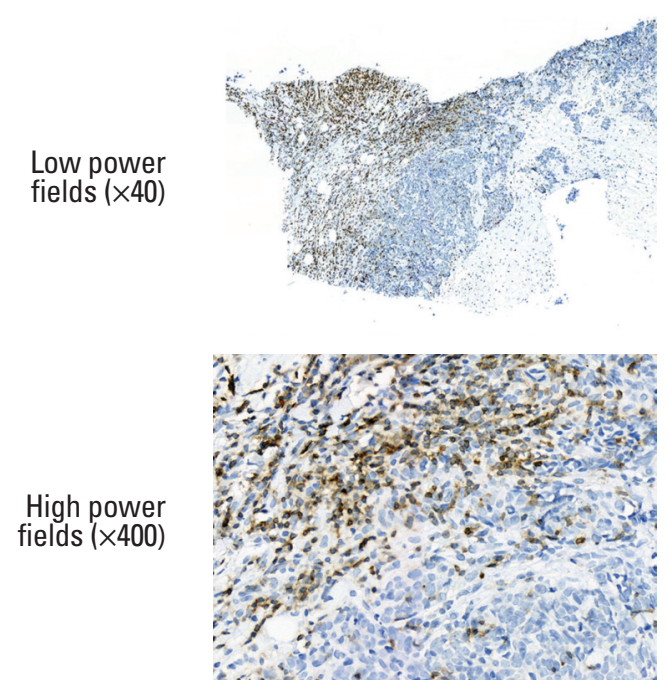

CD8
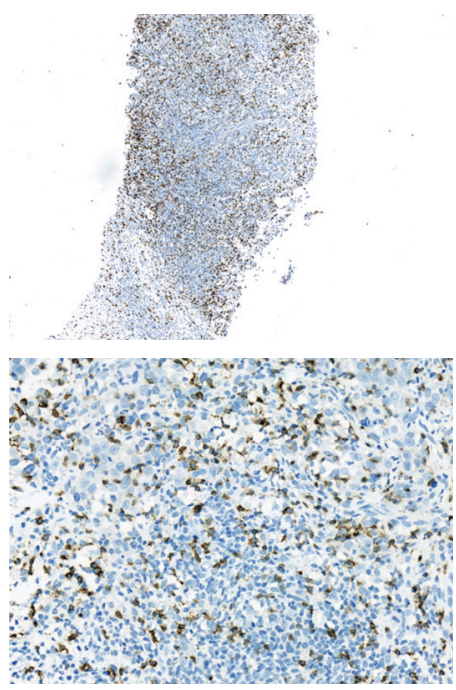

CD20
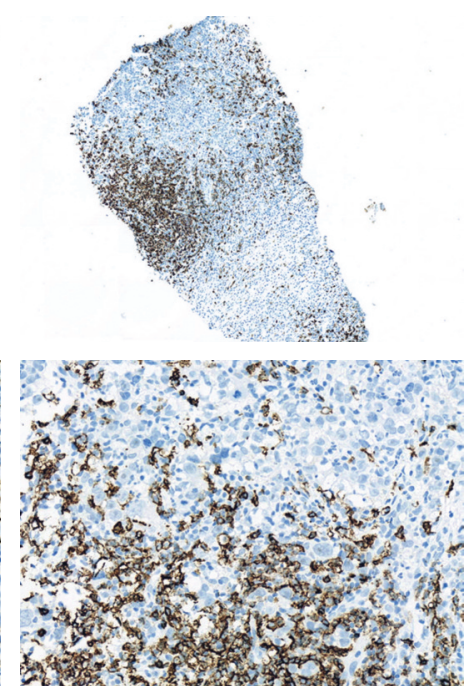

Fig. 2. Representative microscopy images of tumor-infiltrating lymphocyte subpopulations in triple-negative breast cancer sections with immunohistochemical staining.

Table 4. Predictive value for $\mathrm{PCR}$ and the prognostic significance of the protein and gene expression of TIL-associated molecules and HEV density in cases with available FFPE tissue blocks

\begin{tabular}{|c|c|c|c|c|c|c|}
\hline \multirow{2}{*}{ Parameter } & \multicolumn{3}{|c|}{ Predictive value for $\mathrm{pCR}$} & \multicolumn{3}{|c|}{ Disease-free survival } \\
\hline & HR & $95 \% \mathrm{CI}$ & p-value & HR & $95 \% \mathrm{CI}$ & p-value \\
\hline HEV density & 20.325 & $1.455-283.857$ & 0.025 & 0.091 & $0.006-1.421$ & 0.087 \\
\hline CD8-positive cell density & 1.001 & 0.999-1.002 & 0.222 & 0.998 & $0.996-1.000$ & 0.037 \\
\hline CD3-positive cell density & 1.003 & $1.000-1.005$ & 0.052 & 0.999 & $0.997-1.001$ & 0.452 \\
\hline CD20-positive cell density & 1.004 & $1.000-1.007$ & 0.037 & 0.998 & $0.996-1.001$ & 0.194 \\
\hline CXCL13 gene expression & 1.706 & $1.079-2.696$ & 0.022 & 0.634 & $0.478-0.841$ & 0.002 \\
\hline
\end{tabular}

$\mathrm{pCR}$, pathologic complete response; TIL, tumor-infiltrating lymphocyte; $\mathrm{HEV}$, high endothelial venule; FFPE, formalin-fixed paraffin-embedded; HR, hazard ratio; $\mathrm{CI}$, confidence interval.

$20.92 \mathrm{~mm}^{2}$ (range, 1.23 to $83.15 \mathrm{~mm}^{2}$ ). The total number of HEVs in each slide ranged from 0 to 72 . Most of the HEVs were located in the peritumoral areas. The mean MECA79positive HEV density in each slide was $0.169 \pm 0.223 / \mathrm{mm}^{2}$ (Fig. 1). Finally, the mean CD3-, CD8-, and CD20-positive cell densities in the tumor areas of each slide were $357.2 \pm 412.2$ / $\mathrm{mm}^{2}, 402.2 \pm 382.4 / \mathrm{mm}^{2}$, and $246.8 \pm 343.5 / \mathrm{mm}^{2}$, respectively (Fig. 2). The TIL and TLS levels were correlated based on the H\&E-stained slides, the densities of HEV and TIL subpopulations, and expression levels of the CXCL13 mRNA transcripts using the NanoString method with the pathological responses to NAC (Supplementary Table 1). The TLS levels were correlated significantly with the TIL levels, CD3-, CD8-, and CD20-positive cell densities, the HEV density, and expression levels of the CXCL13 mRNA transcripts. The Miller-Payne grade was significantly correlated with the TLS level, CD20-positive cell density, HEV density, and expression levels of CXCL13 mRNA transcripts. Finally, the RCB class was correlated significantly with CXCL13 expression.

\section{Predictive value and prognostic significance of the TIL subpopulations and HEV densities evaluated by IHC}

Based on an analysis of the IHC slides, 17 patients (30.1\%) with available FFPE tissue blocks achieved pCR; none of these patients showed a recurrence during a median follow- 
up period of 31.4 months (range, 21.1 to 53.0 months). Among the patients without pCR $(n=38)$, approximately half had disease recurrence (range of recurrence-free survival, 1.5 to 19.7 months). CD20-positive cell density (HR, 1.004; 95\% $\mathrm{CI}, 1.000$ to $1.007 ; \mathrm{p}=0.037)$, HEV density (HR, 20.325; 95\% CI, 1.455 to 283.857; $\mathrm{p}=0.025)$, and the levels of the CXCL13 mRNA transcripts (HR, 1.706; 95\% CI, 1.079 to 2.696; $\mathrm{p}=0.022$ ) were correlated significantly with $\mathrm{pCR}$. In survival analysis, the CD8-positive cell density and levels of the CXCL13 mRNA transcripts were closely associated with the disease-free survival (Table 4).

\section{Discussion}

To the best of the authors' knowledge, the current study is the first to identify and measure the TLSs in biopsy specimens in an NAC setting, and evaluate their predictive value. Several reports have indicated a prognostic value for the presence of TLSs in cancers [7,11-14]. On the other hand, no studies have assessed this in the context of NAC, which is performed frequently in the treatment of breast cancer. In the present study, both higher levels of TLSs represented by MECA79-positive HEV densities and higher levels of TILs in the H\&E slides were found to be predictors of pCR.

In previous studies, the optimal way to measure the TLSs is controversial. Currently, measurements of TILs are generally performed based on visual assessments of the H\&Estained sections, which is an inexpensive, simple, and versatile method that is often easier to perform than complementary methods, such as IHC [22]. In H\&E-stained tissues, however, it can be difficult to distinguish lymphoid aggregates from true TLSs and it is particularly challenging to quantify the amount of TLSs. In addition, there have been trials in which dendritic cell-lysosomal associated membrane protein (DC-LAMP)-positive dendritic cells were counted as surrogate markers for TLSs [11]. This represents a quantitative and objective method compared to simple visual assessments, even though counting a large number of small dendritic cells by microscopy is quite demanding. In addition, the TLSs show a wide spatial heterogeneity in tumor tissues, and may therefore, be underrepresented when measured in small core needle biopsy specimens or with tissue microarrays [22]. This can be problematic for breast cancers because many breast cancer patients only receive neoadjuvant systemic therapy after a needle biopsy, which represents the only tissue reference available for determining the drug regimens and assessing responses. Previously, the expression of immune-related genes in pre-NAC biopsies obtained from TNBC patients was investigated and the expression of the CXCL13 mRNA transcripts, which encodes a potent B cell-attracting chemokine that contributes to TLS formation in inflammatory and cancerous settings, was found to be a predictor of $\mathrm{pCR}$, which indicates a better prognosis in TNBC patients [15]. On the other hand, it can be easy to under or overestimate gene expression analyses of each biopsy specimen because the proportion of tumor versus normal tissues in a biopsy specimen is not considered. Furthermore, gene expression analysis is expensive and can be cumbersome to perform in daily pathology practice.

Despite these difficulties, the present study obtained some important findings based on MECA79 IHC staining. The number of MECA79-positive HEVs were counted in each slide and the HEV density was calculated. This quantitative method is easier than counting DC-LAMP because of the small number of HEVs. The HEV density was positively correlated with the amount of TLSs measured by assessing the H\&E slides, and a greater HEV density was not only a predictor of $\mathrm{pCR}$, but was also associated with a better diseasefree survival. HEVs contribute to TIL infiltration, and consequently to anti-tumor immunity, so cancer immuntherapy approaches have been proposed, in which the endothelial cell properties are modified to increase immune cell infiltration [24]. Hopefully, the discovery of a method to increase $\mathrm{HEV}$ in the peritumoral areas will be beneficial for the development of treatment strategies for breast cancer, along with many other cancer types.

Finally, the densities of the TIL subpopulations were measured based on IHC staining for CD3, CD8, and CD20, along with a digital microscopic scanner and automated analysis software. Several studies used IHC to evaluate the TIL subpopulations in breast cancer [7,20,25-27]. On the other hand, most of those studies used semiquantitative methods for the measurements because fully quantitative methods are often difficult and time-consuming due to the large number of TILs. Using automated computer software, quantified the cell densities were more accurately and the inter-observer bias was avoided. A higher CD20-positive cell density was found to be a predictor of pCR, whereas a higher CD8-positive cell density was associated with a better disease-free survival. CD8-positive cytotoxic T lymphocytes exhibit antitumor effects via direct tumor cell lysis and interferon $\gamma$ release, which are strongly associated with better therapeutic responses and enhanced patient survival $[17,26]$. In contrast, the effects of B cells on the tumors remain the subject of debate. Some studies have reported that B-cell infiltration is associated with a good prognosis $[20,25]$. The possible mechanisms whereby B cells might mediate improved anti-tumor responses have been suggested, such as the generation of anti-tumor antibodies, the secretion of proimmunogenic cytokines and chemokines, antigen presentation to T cells, and direct cytotoxicity via granzyme B production [28]. Nev- 
ertheless, more study will be needed to explore how the immune components interact in response to tumors.

\section{Conflicts of Interest}

Conflict of interest relevant to this article was not reported.

\section{Conclusion}

The density of MECA79-positive HEV is an easy to assess and accurate surrogate marker of TLS in needle biopsies of TNBC. Moreover, it is positively correlated with pCR in the context of NAC. Overall, approaches to increase HEV may be beneficial in cancer immunotherapy.

\section{Electronic Supplementary Material}

Supplementary materials are available at Cancer Research and Treatment website (http:// www.e-crt.org).

\section{Acknowledgments}

This study was supported by the Basic Science Research Program administered by the National Research Foundation of Korea (NRF) and funded by the Ministry of Science, ICT \& Future Planning (2015R1C1A1A02036484). Gyungyub Gong works as a consultant for Genentech.

\section{References}

1. Aaltomaa S, Lipponen P, Eskelinen M, Kosma VM, Marin S, Alhava E, et al. Lymphocyte infiltrates as a prognostic variable in female breast cancer. Eur J Cancer. 1992;28A:859-64.

2. Clemente CG, Mihm MC Jr, Bufalino R, Zurrida S, Collini P, Cascinelli N. Prognostic value of tumor infiltrating lymphocytes in the vertical growth phase of primary cutaneous melanoma. Cancer. 1996;77:1303-10.

3. Lee HJ, Seo JY, Ahn JH, Ahn SH, Gong G. Tumor-associated lymphocytes predict response to neoadjuvant chemotherapy in breast cancer patients. J Breast Cancer. 2013;16:32-9.

4. Ahn SG, Jeong J, Hong S, Jung WH. Current Issues and clinical evidence in tumor-infiltrating lymphocytes in breast cancer. J Pathol Transl Med. 2015;49:355-63.

5. West NR, Milne K, Truong PT, Macpherson N, Nelson BH, Watson $\mathrm{PH}$. Tumor-infiltrating lymphocytes predict response to anthracycline-based chemotherapy in estrogen receptornegative breast cancer. Breast Cancer Res. 2011;13:R126.

6. Stagg J, Allard B. Immunotherapeutic approaches in triplenegative breast cancer: latest research and clinical prospects. Ther Adv Med Oncol. 2013;5:169-81.

7. Martinet L, Garrido I, Filleron T, Le Guellec S, Bellard E, Fournie JJ, et al. Human solid tumors contain high endothelial venules: association with $\mathrm{T}$ - and B-lymphocyte infiltration and favorable prognosis in breast cancer. Cancer Res. 2011;71: 5678-87.

8. Lee HJ, Kim JY, Park IA, Song IH, Yu JH, Ahn JH, et al. Prog- nostic significance of tumor-infiltrating lymphocytes and the tertiary lymphoid structures in HER2-positive breast cancer treated with adjuvant trastuzumab. Am J Clin Pathol. 2015; 144:278-88.

9. Martinet L, Le Guellec S, Filleron T, Lamant L, Meyer N, Rochaix $\mathrm{P}$, et al. High endothelial venules (HEVs) in human melanoma lesions: major gateways for tumor-infiltrating lymphocytes. Oncoimmunology. 2012;1:829-39.

10. Girard JP, Moussion C, Forster R. HEVs, lymphatics and homeostatic immune cell trafficking in lymph nodes. Nat Rev Immunol. 2012;12:762-73.

11. Ladanyi A, Kiss J, Somlai B, Gilde K, Fejos Z, Mohos A, et al. Density of DC-LAMP(+) mature dendritic cells in combination with activated $\mathrm{T}$ lymphocytes infiltrating primary cutaneous melanoma is a strong independent prognostic factor. Cancer Immunol Immunother. 2007;56:1459-69.

12. Dieu-Nosjean MC, Antoine M, Danel C, Heudes D, Wislez M, Poulot $\mathrm{V}$, et al. Long-term survival for patients with nonsmall-cell lung cancer with intratumoral lymphoid structures. J Clin Oncol. 2008;26:4410-7.

13. Di Caro G, Bergomas F, Grizzi F, Doni A, Bianchi P, Malesci A, et al. Occurrence of tertiary lymphoid tissue is associated with T-cell infiltration and predicts better prognosis in earlystage colorectal cancers. Clin Cancer Res. 2014;20:2147-58.

14. Gu-Trantien C, Loi S, Garaud S, Equeter C, Libin M, de Wind $\mathrm{A}$, et al. CD4(+) follicular helper $\mathrm{T}$ cell infiltration predicts 
breast cancer survival. J Clin Invest. 2013;123:2873-92.

15. Lee HJ, Lee JJ, Song IH, Park IA, Kang J, Yu JH, et al. Prognostic and predictive value of NanoString-based immune-related gene signatures in a neoadjuvant setting of triple-negative breast cancer: relationship to tumor-infiltrating lymphocytes. Breast Cancer Res Treat. 2015;151:619-27.

16. Neyt K, Perros F, GeurtsvanKessel CH, Hammad H, Lambrecht BN. Tertiary lymphoid organs in infection and autoimmunity. Trends Immunol. 2012;33:297-305.

17. Mahmoud SM, Paish EC, Powe DG, Macmillan RD, Grainge MJ, Lee AH, et al. Tumor-infiltrating CD8+ lymphocytes predict clinical outcome in breast cancer. J Clin Oncol. 2011;29: 1949-55.

18. Liu S, Lachapelle J, Leung S, Gao D, Foulkes WD, Nielsen TO. CD8+ lymphocyte infiltration is an independent favorable prognostic indicator in basal-like breast cancer. Breast Cancer Res. 2012;14:R48.

19. Schmidt M, Bohm D, von Torne C, Steiner E, Puhl A, Pilch H, et al. The humoral immune system has a key prognostic impact in node-negative breast cancer. Cancer Res. 2008;68: 5405-13.

20. Brown JR, Wimberly H, Lannin DR, Nixon C, Rimm DL, Bossuyt V. Multiplexed quantitative analysis of CD3, CD8, and CD20 predicts response to neoadjuvant chemotherapy in breast cancer. Clin Cancer Res. 2014;20:5995-6005.

21. Symmans WF, Peintinger F, Hatzis C, Rajan R, Kuerer H, Valero $\mathrm{V}$, et al. Measurement of residual breast cancer burden to predict survival after neoadjuvant chemotherapy. J Clin
Oncol. 2007;25:4414-22.

22. Salgado R, Denkert C, Demaria S, Sirtaine N, Klauschen F, Pruneri G, et al. The evaluation of tumor-infiltrating lymphocytes (TILs) in breast cancer: recommendations by an International TILs Working Group 2014. Ann Oncol. 2015;26:259-71.

23. Geiss GK, Bumgarner RE, Birditt B, Dahl T, Dowidar N, Dunaway DL, et al. Direct multiplexed measurement of gene expression with color-coded probe pairs. Nat Biotechnol. 2008;26:317-25.

24. Bellone M, Calcinotto A. Ways to enhance lymphocyte trafficking into tumors and fitness of tumor infiltrating lymphocytes. Front Oncol. 2013;3:231.

25. Mahmoud SM, Lee AH, Paish EC, Macmillan RD, Ellis IO, Green AR. The prognostic significance of B lymphocytes in invasive carcinoma of the breast. Breast Cancer Res Treat. 2012;132:545-53.

26. Seo AN, Lee HJ, Kim EJ, Kim HJ, Jang MH, Lee HE, et al. Tumour-infiltrating CD8+ lymphocytes as an independent predictive factor for pathological complete response to primary systemic therapy in breast cancer. Br J Cancer. 2013;109:2 705-13.

27. Kang H, Cheong H, Cho MS, Koo H, Han WS, Lee KE, et al. Significance of Foxp3 positive regulatory $\mathrm{T}$ cell and tumor infiltrating $\mathrm{T}$ lymphocyte in triple negative breast cancer. Korean J Pathol. 2011;45:53-61.

28. Nelson BH. CD20+ B cells: the other tumor-infiltrating lymphocytes. J Immunol. 2010;185:4977-82. 